\title{
ACTIVIDAD, EMPLEO Y PARO TURÍSTICO EN LAS REGIONES ESPAÑOLAS (1991-1995)*
}

\author{
Manuel J. Marchena Gómez** \\ Enrique L. Santos Pavón
}

\section{RESUMEN}

El artículo pretende realizar un primer acercamiento al análisis del mercado de trabajo en la actividad turística y su significación territorial y socioeconómica tanto a nivel estatal como regional. Tras un primer planteamiento conceptual y metodológico, se comprueba para las dos escalas citadas el comportamiento claramente positivo que ha tenido el sector turístico —en especial el segmento hotelero - en cuanto al sostenimiento y expansión del empleo entre 1991 y 1995. A pesar de estos datos cuantitativos, se deja entrever cierta preocupación por el crecimiento de la eventualidad, una velada descualificación profesional y el alto grado de estacionalidad en determinadas regiones. En cualquier caso, el artículo examina las diferencias espaciales, a escala regional, de la actividad turística española, mirada desde la perspectiva del mercado de trabajo.

\begin{abstract}
This article seeks an initial approach to analyzing the Tourist Industry labour market and its spacial and socioeconomic significance at a state as well as at a regional level. First of all, concepts and methodology are expounded. Secondly, it is proved that the tourist sector, especially the hotel trade, has had a clearly positive behaviour at the two named scales, because of the steady situation and the expansion of employment between 1991 and 1995. In spite of these quantitative data, there are also some negative factors to be worried about wich are the increase of the temporary work, a veiled decrease of the proffesional training of workers and the high quantity of seasonal work in several regions. Anyway, the article examines the spacial diferences, at a regional scale, of the spanish Tourist Industry, looked from the labour market perspective.
\end{abstract}

* Este trabajo fue seleccionado y presentado como Ponencia en el III Seminario Internacional Impactos Territoriales de los Procesos de Restructuración: Mercados de Trabajo y Desigualdades Sociales, La Rábida (Huelva) 23-25 septiembre 1996.

** Departamento de Geografía Física y Análisis Geográfico Regional. Universidad de Sevilla (España). 


\section{Introducción: magnitudes turísticas y problemática conceptual}

La reciente publicación de la Tabla Insumo-Producto de la Economía Turística española, referida al año de 1992, confirma científicamente aspectos que eran puestos de manifiesto por la evidencia empírica para el conjunto de la economía, la sociedad y el territorio español ${ }^{1}$. Nos referimos básicamente a tres hechos:

(i) Se cuantifica la magnitud y los efectos económicos de la producción turística española medida del lado de la demanda - del consumo de los turistas y sus efectos multiplicadores - y resulta ser que el turismo es nada menos que el 9\% de la producción total (nueve billones de pesetas de producción turística) y del valor añadido bruto (cinco billones de pesetas) de la economía nacional ${ }^{2}$. Algo así como el doble de la producción agrícola y semejante al valor añadido bruto del sector de la construcción en nuestro país. Realmente una participación relativa y bruta determinante en la salud de la economía española ${ }^{3}$.

(ii) Los efectos intersectoriales del turismo son amplios, tanto que puede denominársele como clave y estratégico para la economía nacional ${ }^{4}$. En el sentido de que los impactos económicos del turismo se difunden por el resto del tejido económico español y no se agotan en la satisfacción de la propia demanda turística. El conjunto del sistema económico español produce como efecto multiplicador del turismo, 1,6 pesetas por cada una de demanda propiamente turística. Se pone de relieve la trascendencia de las interrelaciones en muy diversas ramas de actividad — comercio, finanzas, complejo agroindustrial, energía, empresas, construcción...- que origina el turismo en la estructura productiva españo$1 a^{5}$.

(iii) Pero lo que más nos interesa al respecto de este artículo, la inducción de empleo, motivada por la demanda turística, tanto la española ${ }^{6}$ como la extranjera, es según las Tablas Insumo-Producto más arriba citadas, de 1.025.300 ocupaciones directas e indirectas para 1992. Un número que duplica al de sectores como los empleados en la industria química o en el de maquinaria agrícola e industrial. En un país aquejado estructuralmente por el desempleo, desde luego, es una cifra que rondando el 10\% de las ocupaciones, demuestra la magnitud de la actividad turística en España.

En efecto, los datos que manejamos exhiben el trascendente papel del turismo en la economía y el mercado de trabajo nacional ${ }^{7}$. Situándonos precisamente en el aspecto que

1 Tabla Intersectorial de la Economía Turística TIOT 92, Ministerio de Comercio y Turismo. Instituto de Estudios Turísticos, Madrid 1996. La dirección académica del trabajo corresponde a CUADRADO ROURA, J.R y la técnica a FIGUEROLA PALOMO, M.

2 Unos 85.000 millones de dólares y 45.000 millones de dólares, respectivamente, al cambio en pesetas corrientes de 1996.

3 Véase, MARCHENA, M. y VERA, F.: «El modelo turístico español: perspectiva económica y territorial», en Introducción a la Economía del Turismo en España, Ed. Cívitas, Madrid, 1996.

4 Véase, AECIT: La actividad turística española en 1994, Asociación Española de Expertos Científicos en Turismo, Madrid, 1996, p. 575.

5 Con otro elemento digno de mención: la fuga por importaciones originadas por la demanda turística es realmente mínima en comparación con otros sectores económicos en España. Así, según las TIOT 92, sólo un 5\% es importado para satisfacer la demanda turística; por el 56\% en automóviles o el $29 \%$ en el textil.

6 Por otra parte la cuota mayor en la producción turística nacional: 55\%, proporción que nos manifiesta la importancia para el turismo del consumo interno de los españoles.

7 Pueden consultarse los artículos que como complementarios aparecen en la publicación de las Tablas Intersectoriales... (op. cit.), especialmente el de CUADRADO, J.R. y ARRANZ, A.: «La dimensión económica de la actividad turística» (pp. 281-311). 
nos interesa, la actividad, el empleo y el paro creado por el turismo, debe deducirse por consiguiente, el interés estratégico que recae en la producción turística para el mantenimiento de algo tan raro y caro en España como es el empleo ${ }^{8}$.

Verdaderamente, la medición de la producción turística realizada desde el método Insumo-Producto y sus consecuencias sobre la generación de actividad y empleo, directo e indirecto, está hecha desde la concepción que lo que define el producto turístico no son las características intrínsecas del bien o el servicio producido sino el hecho de que sea demandado por un turista (el consumo de los no residentes españoles fuera de su lugar de residencia habitual y de los extranjeros en España por motivos de ocio y turismo). De forma que la cuantificación de producción y empleo es bien diferente a la que se hace convencionalmente desde la contabilidad económica nacional y regional ${ }^{9}$. En este sentido el turismo excede obviamente como complejo productivo a la mera cuantificación del lado de la oferta de la producción hotelera y de restaurantes, y se extiende, por lo menos, a las ramas de viajes, servicios al turista, comercio, intermediación del transporte, inmobiliariaturística, publicidad y otras ${ }^{10}$.

Es por ello que si nos acercamos a investigar la actividad y el empleo turístico en las economías regionales españolas, entendiendo que, por lógica político-administrativa y por razones estadísticas, éstas se delimitan en cuanto Comunidades Autónomas; la aproximación metodológica y de fuentes sea complicada. Escondiéndose en el fondo de la cuestión, el magro reconocimiento social, científico y político que el turismo, paradójicamente, merece en España, especialmente cuando es necesario tomar decisiones «justo a tiempo» en el sistema turístico por la influencia del ciclo corto y la coyuntura. Podríamos decir que el turismo es postfordista antes que el postfordismo ${ }^{11}$, y que en España necesita de un tratamiento más cualitativo para su comprensión como elemento estructurante de las economías nacional y regionales ${ }^{12}$.

Para detectar el empleo turístico y su configuración territorial y profesional se pueden manejar, fundamentalmente ${ }^{13}$, tres caminos estadísticos en España:

8 Sin embargo se deduce teóricamente de los resultados relacionales de la TIOT 92, que sólo son necesarios 5,4 millones de pesetas (unos 50.000 dólares) de demanda turística final para originar un ocupado, ya en el sector turístico, ya en cualquier otros sector económico impactado o inducido por el consumo turístico.

9 Las propuestas de la Organización Mundial del Turismo son precisas en recomendar Cuentas Satélites de producción turística en la contabilidad nacional. Véase, OMT, «Recommendations on tourism statistics», Statistical Papers, ST/ESA/STAT/SER, Madrid 1993, p. 38; y LIBREROS, M.: Cuenta Satélite de turismo, OMT, Madrid 1995 (documento borrador), p. 124.

10 Hemos reflexionado sobre estas cuestiones desde el punto de vista del desarrollo regional en VERA, F. y MARCHENA, M.: «Efectos del turismo en las estructuras regionales periféricas: una aproximación analítica», en Factores de desarrollo en regiones periféricas, XXI Reunión de Estudios Regionales, Vigo, noviembre de 1995 , p. 50.

11 Acaba de defenderse una Tesis Doctoral sobre ello: DONAIRE BENITO, J.A.: El turismo a los ojos del postmodernismo, Universidad Autónoma de Barcelona y Universidad de Girona, 1996, II tomos. Véase, MARCHENA, M.: «Un ejercicio prospectivo: de la industria del turismo fordista al ocio de producción flexible», en Turismo y Desarrollo: nuevos desafíos estructurales (ed. MARCHENA y VERA), Papers de Turisme, $\mathrm{n}^{\circ}$ 14-15, Valencia 1994, pp. 77-95.

12 BOTE, V.: «Estructura y Desarrollo del Turismo en España: hacia un cambio cualitativo y más responsable», en $V$ Congreso Nacional de Economía sobre Economía de los Servicios, Las Palmas de Gran Canaria, diciembre de 1995 , p. 35.

13 No es posible encontrar, en las fuentes existentes a nuestro alcance, datos sobre actividad y empleo en el sector turístico en todas sus dimensiones, puesto que no es considerado como un sector en sí mismo. Aparte de las fuentes descritas sucintamente en el texto, podrían mencionarse también los Censos de Población, y especialmente, la Encuesta sobre la Estructura de los Establecimientos Hoteleros, que sólo se realiza quinquenalmente, y la Distribución Salarial en España, publicada por última vez en 1992. Todas fuentes, del Instituto Nacional de Estadística español, que tienen dificultades obvias para el análisis diacrónico y son insuficientes por la parcialidad de sus planteamientos con respecto a la actividad turística. 
A) Por el método reflejado de la valoración de empleo a través de Tablas InsumoProducto como la antes citada, con las carencias inherentes a la falta de series y el excesivo componente estimativo y de coeficientes relacionales que comporta la construcción de la tabla. De todos modos, consideramos que es un instrumento insustituible para la aprehensión del turismo del lado de la demanda y de su impacto en el empleo requerido para satisfacer dicha demanda ${ }^{14}$; incluso con posibilidades de regionalizar los resultados tanto de producción como de empleo ${ }^{15}$.

B) A través del Movimiento de viajeros en establecimientos hoteleros (MVEH, Instituto Nacional de Estadística - INE-), donde se recogen, entre otras muchas variables, series de empleados declarados por los empresarios contratados exclusivamente en el sector considerado de forma clásica como turístico: el hotelero oficialmente reglado. Por tanto se restringe de forma decisiva la cuantificación de la ocupación turística por cuanto se desprecia no sólo ramas afines a la actividad turística, sino todo el universo extrahotelero de alojamientos que precisamente en España representa aproximadamente el 80\% de la oferta ${ }^{16}$. En cualquier caso su seguimiento serial y temporal nos proporciona un buen indicador relativo del estado tendencial del empleo turístico en España.

C) Por medio de la Encuesta de Población Activa (EPA, también elaborada por el INE); es evidente que no se trata de una fuente especialmente turística como la anterior, pero con todas sus deficiencias nos ayuda a aproximarnos de forma más coherente a la complejidad del fenómeno estudiado. En efecto, la segmentación por ramas de actividad de la EPA, permite la agregación para configurar la ocupación turística de manera más cercana a la realidad; en este caso del lado de la oferta. Así, hemos convenido por las limitaciones estadísticas, que la actividad turística sería exclusivamente - decisión también parcial, por impoderables estadísticos- la suma de los activos, los empleos, y los parados de las ramas de hostelería y restauración y la de las actividades anexas a los viajes: agencias de viajes; en la serie elegida 1991-1995 ${ }^{17}$.

Como se verá falta actividad, directa pero sobre todo indirecta, inducida por la demanda turística en esta opción, pero también parte de la actividad y la ocupación de los subsectores mencionados no se dedica necesariamente a satisfacer la demanda turística. Hemos realizado una comprobación simple que acerca la calidad de este sistema con las magnitudes de empleo que arroja la Tabla Intersectorial citada. A saber, la Encuesta de Población Activa para el mismo año (1992) al que se refiere la Tabla, por el procedimiento comentado, nos dice que en España existían 899.000 empleos turísticos; la Tabla estima 850.000 ocupados en las ramas típicamente turísticas, prácticamente las que hemos selec-

14 Es decir, la Tabla no hace referencia al número de puestos de trabajos creados, sino a requerimientos de empleo proporcionales a los coeficientes resultantes de la estructura productiva estimada en la TIOT-92.

15 Método propuesto por FIGUEROLA, M.: «Regionalización de la renta turística de España (base TIOT)», en Tabla Intersectorial... (op. cit.), pp. 333-351.

16 Según hemos demostrado en VERA, F. y MARCHENA, M.: «Promoción Inmobiliaria y Configuración de la Oferta Turística», en Marché inmobilier et développement touristique, AIEST, vol. 37, St. Gall (Suiza) 1995, pp. 29-53.

17 Se ha elegido el primer quinquenio de los noventa, por dos razones esencialmente: (i) porque se detecta un cambio de ciclo turístico capital en el interior del mismo, a saber, de la bonanza de finales de los ochenta se produce la recesión turística de inicios de los noventa y la euforia turística —año histórico- desde 1994; y (ii) porque entre 1992-1993 se aprueba el cambio en la legislación laboral española, flexibilizando y desregulando el mercado de trabajo. Comprobaremos que el empleo turístico es extraordinariamente flexible y se ajusta con total ductilidad al ciclo. Hecho que corrobora agudamente para Andalucía, AURIOLES, J.: «Turismo y Empleo en Andalucía. Una interpretación del cambio de los niveles de productividad en el sector de la hostelería», en II Foro del Turismo de Andalucía, Consejería de Turismo y Deportes, Granada 1996, 19 ff. 
cionado en la EPA. Sin embargo cuando se establecen los ocupados directos a partir de la demanda turística la cifra baja en la Tabla a 633.000 y se eleva a 1.250 .300 de ocupados ya mencionados, cuando se agregan además de los de impacto directo, los ocupados indirectamente por mor del consumo de los turistas. Magnitudes que consideramos conformes para calibrar el peso del empleo turístico en España, del lado de la oferta y del lado de la demanda respectivamente por la horquilla cuantitativa en la que se ubican.

\section{Caracterización general del mercado de trabajo turístico entre los años 1991 y 1995}

El marco general del mercado de trabajo español en los primeros años de los 90 se caracteriza básicamente por tres hechos:

- un lento aumento de la población activa, que crece a un ritmo del $1 \%$ anual en el período de estudio, fruto de la maduración del régimen demográfico español y el cese de anteriores procesos expansivos (cuadro 1),

- un paulatino descenso del número de ocupados, que se contrae un 4,5\% entre 1991 y 1995, consecuencia fundamental de la crisis económica general de principios de los 90 y que sufren especialmente sectores como la agricultura y la industria ${ }^{18}$,

- un progresivo aumento del número de parados entre 1991 y 1994, con una leve recuperación en el 95, año en el que ya se vislumbra una débil pero segura recuperación de la actividad económica y el empleo, tal y como lo demuestra el hecho de que por primera vez en lo que va de década se recupera el empleo, con una tasa de crecimiento del 2,6\% respecto al año anterior (cuadro 3).

El Sector Servicios ofrece unos datos esclarecedores acerca de su dinamismo y resultados positivos dentro de la estructura económica general, ya que, aunque en los años más difíciles del período (1992 y 1993) descendiese el número de ocupados en el mismo, la recuperación posterior es fuerte, muy superior a la dinámica negativa mantenida en la agricultura y la industria o a la leve recuperación de la construcción ${ }^{19}$.

Esa dinámica expansiva del empleo en el sector servicios, que demuestra su contribución positiva al mercado de trabajo frente a la alarmante contracción de otros sectores de actividad, no debe llevarnos a engaño en el sentido de concebir un crecimiento homogéneo y por igual de todos los subsectores, sino que se observan claras diferencias internas entre ramas de actividad ${ }^{20}$.

Las actividades turísticas presentan una perfecta adecuación a las tendencias generales de la actividad y el empleo, aunque con mejores resultados tanto en tiempos de recesión como en los de recuperación ${ }^{21}$. Esta característica refuerza la hipótesis de que el turismo es

18 Véase cuadro 2. En el caso de la industria la reducción de la población ocupada en el quinquenio es de un $18 \%$, con una destrucción de más de 400.000 puestos de trabajo, siendo especialmente negativo el año 93; la agricultura, por su parte, tiene unas pérdidas de ocupación del 18\%, con un descenso de 240.000 empleos.

19 Véase cuadro 2. La construcción aumenta en 75.000 empleos entre el año 94 y 95 , frente a los casi 250.000 empleos que se crean en el sector servicios.

20 La EPA muestra cómo la recuperación en el 95 de las ramas de hostelería y restauración y de agencias de viajes es superior a la del transporte aéreo o los servicios personales, e inferior a la actividad inmobiliaria, por citar tres ejemplos.

21 Véase cuadro 2. Mientras la población ocupada total descendía en los años 92, 93 y 94 con índices a veces superiores al $4 \%$ y se recuperaba lentamente en el 95 en un $2 \%$, los ocupados en hostelería y agencias de viajes nunca sobrepasarán pérdidas por encima del 2,5\% y disfrutarán de una fuerte recuperación ya en el 94 y continuando en el 95 , con porcentajes del 6,62 y el 6,46\% respectivamente. 
Cuadro 1

EVOLUCIÓN DE LA POBLACIÓN ACTIVA POR SECTORES ECONÓMICOS Y EN

LAS ACTIVIDADES RELACIONADAS CON EL TURISMO* ENTRE 1991 Y 1995

\begin{tabular}{|lrrrrr|}
\hline & \multicolumn{1}{c}{$\mathbf{1 9 9 1}$} & $\mathbf{1 9 9 2}$ & \multicolumn{1}{c}{$\mathbf{1 9 9 3}$} & \multicolumn{1}{c}{$\mathbf{1 9 9 4}$} & \multicolumn{1}{c|}{1995} \\
\hline Activos & 15.073 .100 & 15.154 .800 & 15.318 .800 & 15.468 .200 & 15.625 .400 \\
Agricultura & 1.541 .300 & 1.445 .800 & 1.410 .400 & 1.375 .300 & 1.350 .600 \\
Industria & 3.215 .400 & 3.177 .700 & 3.060 .200 & 2.957 .600 & 2.864 .100 \\
Construcción & 1.519 .400 & 1.537 .900 & 1.530 .000 & 1.474 .200 & 1.474 .100 \\
Servicios & 7.815 .300 & 7.973 .000 & 8.118 .400 & 8.238 .000 & 8.422 .700 \\
Actividades turísticas & 895.600 & 899.400 & 937.100 & 993.000 & 1.026 .800 \\
\hline
\end{tabular}

Fuente: INE. EPA 1991-1995.

* Consideramos como Actividades relacionadas con el turismo a la Hostelería y las Agencias de Viajes.

Cuadro 2

EVOLUCIÓN DE LA POBLACIÓN OCUPADA POR SECTORES ECONÓMICOS Y EN LAS ACTIVIDADES RELACIONADAS CON EL TURISMO* ENTRE 1991 Y 1995

\begin{tabular}{|lrrrrr|}
\hline & \multicolumn{1}{c}{$\mathbf{1 9 9 1}$} & $\mathbf{1 9 9 2}$ & \multicolumn{1}{c}{$\mathbf{1 9 9 3}$} & \multicolumn{1}{c}{$\mathbf{1 9 9 4}$} & \multicolumn{1}{c|}{$\mathbf{9 9 5}$} \\
\hline Ocupados & & & & & \\
Agricultura & 12.609 .400 & 12.366 .200 & 11.837 .500 & 11.730 .100 & 12.041 .900 \\
Industria & 1.345 .100 & 1.252 .700 & 1.197 .700 & 1.150 .900 & 1.106 .100 \\
Construcción & 2.890 .200 & 2.804 .200 & 2.539 .800 & 2.473 .700 & 2.486 .100 \\
Servicios & 1.273 .500 & 1.196 .300 & 1.088 .500 & 1.058 .700 & 1.134 .500 \\
Actividades turísticas & 7.100 .000 & 7.113 .000 & 7.011 .400 & 7.046 .800 & 7.315 .200 \\
& 761.100 & 747.900 & 738.300 & 787.200 & 838.100 \\
\hline
\end{tabular}

Fuente: INE. EPA 1991-1995.

* Consideramos como Actividades relacionadas con el turismo a la Hostelería y las Agencias de Viajes.

Cuadro 3

PARADOS QUE HAN TRABAJADO ANTERIORMENTE POR SECTORES DE ACTIVIDAD Y EN LA RAMA DE HOSTELERÍA 1991-1995

\begin{tabular}{|lrrrrr|}
\hline & \multicolumn{1}{c}{1991} & \multicolumn{1}{c}{1992} & \multicolumn{1}{c}{1993} & 1994 & 1995 \\
\hline Total & 1.841 .500 & 2.164 .400 & 2.787 .200 & 2.937 .800 & 2.769 .900 \\
Agricultura & 196.200 & 193.100 & 212.500 & 224.400 & 244.500 \\
Industria & 325.300 & 373.500 & 520.300 & 483.900 & 378.100 \\
Construcción & 245.900 & 341.600 & 441.500 & 415.600 & 339.600 \\
Servicios & 714.500 & 860.000 & 1.107 .100 & 1.191 .200 & 1.107 .500 \\
Hostelería & 121.900 & 142.900 & 181.700 & 187.000 & 173.900 \\
\hline
\end{tabular}

Fuente: INE. EPA 1991-1995. 


\begin{tabular}{|c|c|c|c|c|c|}
\hline & 1991 & 1992 & 1993 & 1994 & 1995 \\
\hline Total & 37,2 & 36,8 & 36,6 & 36,8 & 36,7 \\
\hline Agricultura & 42,1 & 42,6 & 42,6 & 42,9 & 42,7 \\
\hline Industria metalmecánica & 36,3 & 36,0 & 36,0 & 36,5 & 36,7 \\
\hline Construcción & 37,1 & 36,8 & 36,8 & 36,8 & 36,9 \\
\hline Transportes & 38,6 & 38,1 & 38,1 & 38,4 & 35,7 \\
\hline Intermediación financiera & 36,2 & 36,0 & 36,0 & 35,8 & 41,5 \\
\hline Hostelería & 39,7 & 39,5 & 39,5 & 42,5 & 38,3 \\
\hline
\end{tabular}

Fuente: INE. EPA 1991-1995.

un sector de sostenimiento del empleo en momentos de crisis y de dinamización en situaciones favorables, características que unidas a su flexibilidad y a sus posibilidades de crecimiento futuro hacen del mismo una actividad estratégica para las economías y los mercados de trabajo regionales.

Con respecto al sector servicios considerado globalmente, las actividades turísticas mantienen una dinámica similar en relación a la ocupación, aunque en este caso, a diferencia de la comparación establecida anteriormente con la actividad económica en general, las tendencias del empleo en el turismo son siempre más extremas que las de los servicios en general. Esto quiere decir que la reducción del empleo en tiempos de crisis es mayor y que la expansión del mismo en etapas de crecimiento también lo es, lo cual significa que las actividades turísticas son muy sensibles a la coyuntura económica general y se ajustan fielmente a la misma agudizando su tendencia.

Esta flexibilidad contrasta con la relativa rigidez de los servicios públicos, que por ello muestran menores márgenes de expansión o reducción del empleo. De lo dicho hasta ahora se puede deducir que las actividades turísticas manifiestan señales muy optimistas en cuanto al anhelo general de la reducción del desempleo. Es difícil realizar un análisis diacrónico de los resultados en el período estudiado por motivos metodológicos ${ }^{22}$, pero sí podemos esbozar una tendencia altamente positiva desde del año 93 al 95, con resultados netamente mejores en el desempleo turístico que en las cifras generales ${ }^{23}$.

Desde una perspectiva más cualitativa, analizaremos la caracterización del empleo según el nivel socioprofesional de los trabajadores. En este sentido se puede apreciar que la actividad hostelera presenta un alto porcentaje de directivos (superior a la media del total de actividades, el comercio o las actividades financieras) y una escasa presencia de profesionales, técnicos y personal científico ${ }^{24}$. El personal de servicios supone más de la mitad

22 Véase cuadro 2. Hasta el año 92 incluido, la EPA agregaba los datos de comercio, hostelería y reparaciones, lo cual hace difícil el análisis de las actividades hosteleras en esos años. No obstante se puede hacer una desagregación, tal y como nosotros hemos efectuado en el cuadro 2, tomando como punto de referencia los años posteriores y el peso relativo de cada subsector.

23 Ver cuadro 3. Mientras el número de parados totales, hayan trabajado o no anteriormente, ha aumentado en una $50,4 \%$, los de la hostelería lo han hecho en un $40 \%$.

24 Véanse cuadros 5, 6, 7 y 8. El porcentaje de directivos en el 95 es el mayor de todas las ramas de actividad con un $24 \%$ sobre el total de empleados, frente al $8,3 \%$ de media; los técnicos y profesionales científicos son solamente un $0,3 \%$, la cifra más baja de todas las ramas, que tienen una media del 10,2\%. 
Cuadro 5

TODAS LAS ACTIVIDADES

\begin{tabular}{|lrrrrrr|}
\hline & $\mathbf{1 9 9 3}$ & $\%$ & $\mathbf{1 9 9 4}$ & \multicolumn{1}{c}{$\%$} & $\mathbf{1 9 9 5}$ & $\%$ \\
\hline Total & 11.837 .500 & 100 & 11.730 .100 & 100 & 12.041 .900 & 100 \\
Profesionales y técnicos & 1.468 .800 & 12,4 & 1.150 .200 & 9,8 & 1.222 .900 & 10 \\
Directivos & 234.900 & 2 & 961.600 & 8,2 & 1.009 .100 & 8,3 \\
Administración & 1.623 .700 & 13,7 & 1.274 .600 & 10,8 & 1.214 .800 & 10,0 \\
Personal de servicios & 1.720 .000 & 14,5 & 1.622 .500 & 13,8 & 1.672 .900 & 13,9 \\
No cualificados & & & 1.686 .600 & 14,3 & 1.740 .300 & 14,4 \\
\hline
\end{tabular}

Cuadro 6

HOSTELERÍA

\begin{tabular}{|lrrrrrr|}
\hline & 1993 & $\%$ & 1994 & $\%$ & 1995 & $\%$ \\
\hline Total & 656.200 & 100 & 708.800 & 100 & 755.500 & 100 \\
Profesionales y técnicos & 4.800 & 0,73 & 2.600 & 0,3 & 2.300 & 0,3 \\
Directivos & 182.700 & 24,8 & 186.300 & 26,2 & 181.400 & 24,0 \\
Administración & 31.500 & 4,8 & 34.900 & 4,9 & 34.300 & 4,5 \\
Personal servicios & 385.189 & 58,7 & 389.000 & 54,8 & 427.300 & 56,5 \\
No cualificados & & & 68.600 & 9,6 & 78.400 & 10,3 \\
\hline
\end{tabular}

Cuadro 7

TRANSPORTE Y COMUNICACIONES

\begin{tabular}{|lrrrrrr|}
\hline & 1993 & $\%$ & \multicolumn{1}{c}{1994} & \multicolumn{1}{c}{$\%$} & \multicolumn{1}{c}{1995} & $\%$ \\
\hline Total & 694.900 & 100 & 680.900 & 100 & 724.700 & 100 \\
Profesionales y técnicos & 27.200 & 3,9 & 15.100 & 2,2 & 17.500 & 2,4 \\
Directivos & 12.000 & 1,7 & 21.900 & 3,2 & 25.300 & 3,5 \\
Administración & 191.600 & 27,6 & 150.700 & 22,1 & 156.000 & 21,5 \\
Personal servicios & 19.900 & 2,9 & 19.100 & 2,8 & 23.200 & 3,2 \\
No cualificados & & & 49.200 & 7,2 & 48.900 & 6,8 \\
\hline
\end{tabular}

\section{Cuadro 8}

INTERMEDIACIÓN FINANCIERA

\begin{tabular}{|lrrrrrr|}
\hline & 1993 & $\%$ & 1994 & $\%$ & 1995 & $\%$ \\
\hline Total & 336.300 & 100 & 312.700 & 100 & 315.200 & 100 \\
Profesionales y técnicos & 24.400 & 7,2 & 20.000 & 6,4 & 18.900 & 6,0 \\
Directivos & 43.500 & 12,9 & 31.600 & 10,1 & 38.600 & 12,2 \\
Administración & 217.800 & 64,8 & 183.000 & 58,5 & 169.000 & 53,6 \\
Personal servicios & 13.100 & 3,9 & 800 & 0,3 & 600 & 0,2 \\
No cualificados & & & 13.500 & 4,3 & 10.100 & 3,2 \\
\hline
\end{tabular}

Fuente: INE. EPA 1993-1995.

* No podemos disponer de los datos de 1991 y 1992 porque a partir del año 1993 se utilizará una tipología de niveles socioprofesionales totalmente nueva, que no es compatible con la estructuración anterior. 
de los empleados, muy por encima del personal no cualificado, tendencia inversa a la de las actividades citadas anteriormente, en las que los trabajadores no cualificados superan ampliamente al personal de servicios ${ }^{25}$.

Estos datos diseñan una empresa hostelera tipo donde tienen peso las actividades decisionales y organizacionales, pero no la suficiente importancia el asesoramiento técnico y científico de profesionales con capacidad de innovación y mejora cualitativa de los procesos productivos. Del mismo modo hay que poner en cuarentena la información sobre la cualificación de los trabajadores, ya que aunque los datos hablen de menores tasas de descualificación en las empresas hoteleras que en el resto de sectores, podría verse enmascarada la realidad bajo la sospechosa etiqueta de «personal de servicios», que en muchos de los casos de la actividad que nos ocupa está claramente conformado por personal no cualificado.

En último lugar destacaremos el dato del número medio de horas semanales realizadas por los ocupados según el tipo de actividad. Es significativo el hecho de que la hostelería presente las medias más altas ${ }^{26}$, sólo superada por la agricultura, y que estas aumenten a medida que desciende el número de ocupados y viceversa, lo cual puede traslucir dos hipótesis que a continuación se plantean: el mayor grado de sobreexplotación de los trabajadores de este sector, que en muchos casos realizan servicios de tipo personal más costosos desde el punto de vista de la dedicación temporal, y la menor productividad de su trabajo, puesto que necesitan emplear más horas de trabajo para el sostenimiento de la actividad empresarial.

\section{Turismo y empleo en las regiones españolas entre 1991 y $1995^{27}$}

Las tendencias estatales en cuanto a actividad, ocupación y empleo diseñan una estructura global del mercado de trabajo que es reproducida en mayor o menor medida por las diferentes CC.AA. españolas, dependiendo de sus características estructurales, sus condicionantes coyunturales y sus peculiaridades empresariales, sociolaborales y productivas.

La estabilización de la tasa de actividad estatal entre los años 1991 y 1995 tiene su fiel reflejo en la mayoría de las regiones españolas, aunque siempre se observan desviaciones respecto a la media nacional, que son de carácter expansivo en Comunidades demográficamente jóvenes o de tipo inmigratorio como Andalucía y Madrid, respectivamente, y de carácter constrictivo en regiones deprimidas o en declive (Galicia, Canarias o Asturias).

La interpretación espacial del empleo en España continúa reflejando una situación de fuertes contrastes y desequilibrios interterritoriales. Frente a un grupo de regiones bien posicionadas, con tasas de empleo superiores al 40\% (Baleares, Valencia, Navarra o Cataluña), encontramos en el polo opuesto una serie de espacios regionales que a duras penas mantienen tasas en torno al 30\%, y que son sin duda CC.AA. con grandes dificultades en sus estructuras socioeconómicas, bien sea por razones de atraso histórico y periferismo económico, bien por problemas de declive industrial ${ }^{28}$.

25 Véanse cuadros 5, 6, 7 y 8. El personal de servicios en el 95 supone un 56,5\% en hostelería frente a la media del $13,9 \%$, y los trabajadores no cualificados un $10,3 \%$ frente al $14,4 \%$ de media.

26 Véase cuadro 4. Las medias semanales en hostelería están en torno a las 40 horas, superadas en los años 93, 94 y 95, frente a las 36,8 de media del total de actividades.

27 Para la realización del análisis territorial manejaremos los datos explotados por la Encuesta de Población Activa (EPA) y por el Movimiento de Viajeros en Establecimientos Hoteleros (MVEH), que, como ya se decía en la introducción, son las fuentes básicas para el estudio del mercado de trabajo turístico en las diferentes CC.AA. españolas.

28 Véase mapa 1. Las regiones con tasas de empleo inferiores al 35\% son Andalucía, Extremadura, CastillaLeón (regiones periféricas) y Asturias (región en declive industrial). 
Las tasas de paro ofrecen una lectura muy parecida y directamente relacionada con lo expresado hasta el momento: un conjunto de regiones con tasas claramente inferiores a la media nacional, entre el 10 y el $20 \%$, que se sitúan en la vertiente nororiental de la península (Cataluña, Navarra, Rioja, Aragón), más Baleares y Galicia; otro grupo con cifras en torno a la media y que coinciden básicamente con las regiones del interior, el Levante, la cornisa cantábrica y Canarias; y finalmente, los casos extremos de Andalucía y Extremadura, con tasas alarmantes del 33,91 y el 30,58\% respectivamente ${ }^{29}$.

La panorámica general se completa con un breve análisis de los datos de ocupación en el sector servicios, dentro del cual se sitúa el subsector objeto de nuestro estudio. La tesis de que el sector servicios es el motor fundamental de la creación de empleo en nuestro país ante el retraimiento de los sectores tradicionales, se comprueba de forma indiscutible al analizar los datos sobre la evolución de la ocupación en general y los de la ocupación del sector servicios por CC.AA. entre los años 1991 y 1995. Mientras el número de ocupados desciende en todas las regiones excepto Baleares y Canarias (cuadro 9), los ocupados en el sector servicios aumentan en casi todas las Comunidades, exceptuando aquéllas con mayores problemas económico-productivos y donde ni tan siquiera el sector servicios se salva de la crisis general (cuadro 10).

De las cifras se deduce que sólo las CC.AA. de Canarias y Baleares, por cierto las más evidentemente turísticas, tienen tendencias positivas de empleo entre los años 1991 y 1995, lo cual dice mucho en favor de esta actividad como válvula de escape ante situaciones críticas de destrucción de empleo.

También se observa en las regiones con evidente peso sociolaboral del sector servicios en general (cuadro 11 y mapas 3 y 4 ) y de la actividad turística en particular (mapas 5 y 6 ), un fuerte incremento de los ocupados en el terciario, muy por encima de la media de las demás regiones, con tasas de crecimiento entre 1991 y 1995 del 16,4\% en Baleares, 7,2\% en Canarias, 6,1\% en Andalucía o 5,5\% en Cataluña.

El estudio concreto del mercado de trabajo en la actividad turística lo realizaremos a partir de los datos sobre personal empleado en establecimientos hoteleros ${ }^{30}$, que aunque no aportan una información completa y dimensionada sobre la compleja realidad de la actividad, sí ofrece una idea aproximada y avalada desde el punto de vista científico sobre el peso del empleo y las tendencias del mercado de trabajo turístico en las economías regionales españolas.

Las cifras nos muestran un volumen global de 112.000 empleos en establecimientos hoteleros para el año 1994, de los cuales el $80 \%$ se localiza en seis CC.AA. (Andalucía, Baleares, Canarias, Cataluña, C. Valenciana y Madrid) y el 70\% en el litoral mediterráneo y las islas. De entre todas las regiones destaca Baleares con 20.500 puestos de trabajo, seguida por Andalucía con 18.000 y Canarias con 16.000. Entre las tres suponen el 50\% del empleo en el sector hotelero español (mapa 5).

Estos montantes globales, que ponen de manifiesto las magnitudes territoriales del empleo turístico español (cuadro 12), han de ser completados con un análisis más detallado que nos ofrezca la importancia relativa de ese empleo dentro de la estructura regional del mercado de trabajo. Los datos nos muestran que sólo hay 3 Comunidades en las que el

29 Véase mapa 2. Un análisis más profundo sobre el tema lo encontramos en CARAVACA BARROSO, I. y SÁNCHEZ LECHUGA, P.: «Cambios socioeconómicos, desempleo y desequilibrios territoriales en España», en Revista de Estudios Regionales, $\mathrm{n}^{\circ}$ 42, pp. 15-42. Sevilla: Universidades de Andalucía, 1995.

30 Este es el dato que ofrece el $M V E H$, por CC.AA. y por meses, diferenciando entre personal fijo y eventual. La EPA sólo ofrece datos sobre empleo en la rama de comercio y hostelería, siendo compleja la desagregación de los datos debido al diferente peso que cada subsector tiene en las distintas regiones. 


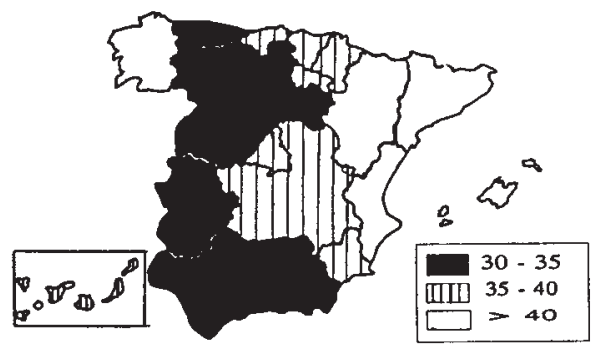

Fuente: INE. EPA 1995. Elaboración propia.

Mapa 2. Tasa de paro 1995 (\%).

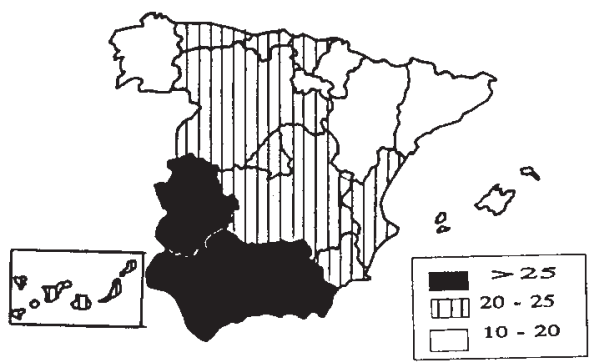

Fuente: INE. EPA 1995. Elaboración propia.

MAPA 3. Ocupados en sesctor servicios 1995 (miles).

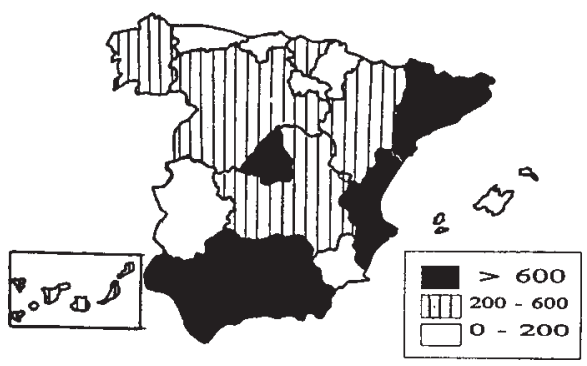

Fuente: INE. EPA 1995. Elaboración propia.

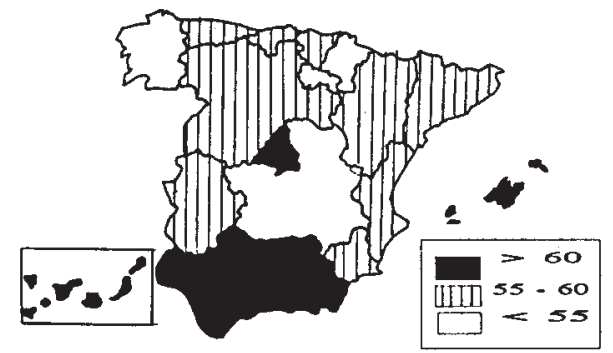

Fuente: INE. EPA 1995. Elaboración propia.

MAPA 5. Empleados en establecimientos

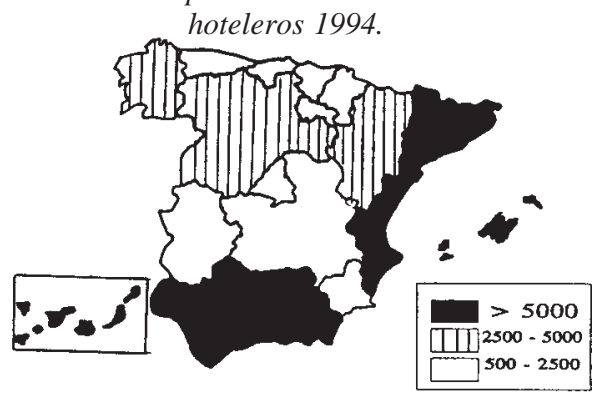

Fuente: INE. EPA 1995. Elaboración propia.

MAPA 6. Empleados en hoteles sobre el total de ocupados en servicios 1994 (\%).

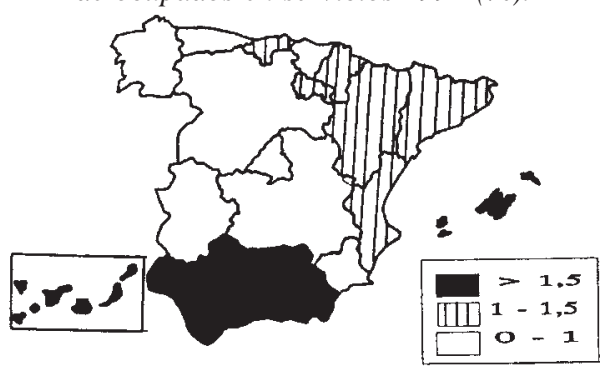

Fuente: INE. EPA 1995. Elaboración propia.

empleo en establecimientos hoteleros tiene un peso relativo importante: Baleares, Canarias y Andalucía (mapas 6 y 7).

El caso de Baleares es el más evidente, con una tasa de ocupados en establecimientos hoteleros del 8,41\% respecto al total de ocupados y del 11,79\% respecto a los ocupados en servicios. Canarias le sigue con unas tasas de 3,5 y 4,62\% respectivamente, y Andalucía aparece destacada sobre el resto de regiones con unas cifras de 1,05 y $1,65 \%$ respectivamente. En el resto de regiones, los ocupados en establecimientos hoteleros no sobrepasan el $1 \%$ del total de ocupados, ni el 1,5\% de los empleados en el sector servicios, aunque hay regiones como Cataluña y Valencia que se acercan a esos parámetros. 
Cuadro 9

EVOLUCIÓN DEL NÚMERO DE OCUPADOS ENTRE 1991 Y 1995

\begin{tabular}{|lrrrrr|}
\hline & \multicolumn{1}{c}{$\mathbf{1 9 9 1}$} & $\mathbf{1 9 9 2}$ & \multicolumn{1}{c}{$\mathbf{1 9 9 3}$} & $\mathbf{1 9 9 4}$ & \multicolumn{1}{c|}{$\mathbf{9 9 5}$} \\
\hline Total & 12.609 .400 & 12.366 .200 & 11.837 .500 & 11.730 .100 & 12.041 .900 \\
Andalucía & 1.834 .100 & 1.802 .000 & 1.696 .700 & 1.688 .200 & 1.731 .500 \\
Aragón & 412.300 & 407.900 & 390.200 & 389.200 & 395.900 \\
Asturias & 351.200 & 342.100 & 323.200 & 307.600 & 311.300 \\
Baleares & 252.900 & 235.400 & 230.500 & 243.300 & 263.900 \\
Canarias & 446.400 & 441.100 & 424.200 & 447.600 & 471.800 \\
Cantabria & 162.300 & 163.300 & 160.400 & 153.900 & 156.400 \\
Castilla-León & 841.600 & 813.900 & 783.700 & 759.800 & 753.700 \\
Castilla-La Mancha & 528.300 & 518.900 & 497.700 & 483.200 & 468.000 \\
Cataluña & 2.223 .000 & 2.163 .300 & 2.051 .200 & 2.052 .700 & 2.127 .600 \\
Valencia & 1.276 .600 & 1.248 .400 & 1.202 .800 & 1.208 .700 & 1.268 .700 \\
Extremadura & 302.600 & 296.300 & 283.500 & 271.000 & 267.700 \\
Galicia & 1.012 .900 & 974.400 & 948.500 & 916.200 & 918.100 \\
Madrid & 1.636 .000 & 1.650 .900 & 1.582 .500 & 1.547 .300 & 1.623 .200 \\
Murcia & 317.000 & 312.600 & 305.000 & 308.200 & 316.700 \\
Navarra & 183.900 & 180.100 & 172.000 & 174.700 & 179.800 \\
País Vasco & 708.500 & 697.200 & 665.000 & 664.900 & 670.300 \\
La Rioja & 90.300 & 86.300 & 85.100 & 82.200 & 84.600 \\
\hline
\end{tabular}

Fuente. INE. EPA 1991-1995.

Cuadro 10

EVOLUCIÓN DE LOS OCUPADOS EN EL SECTOR SERVICIOS ENTRE 1991 Y 1995

\begin{tabular}{|lrrrrr|}
\hline & \multicolumn{1}{c}{$\mathbf{1 9 9 1}$} & $\mathbf{1 9 9 2}$ & \multicolumn{1}{c}{$\mathbf{1 9 9 3}$} & $\mathbf{1 9 9 4}$ & \multicolumn{1}{c|}{$\mathbf{1 9 9 5}$} \\
\hline Total & 7.100 .700 & 7.113 .000 & 7.011 .400 & 7.046 .800 & 7.315 .200 \\
Andalucía & 1.064 .800 & 1.097 .300 & 1.084 .600 & 1.079 .600 & 1.130 .400 \\
Aragón & 216.600 & 216.400 & 213.500 & 211.000 & 220.100 \\
Asturias & 179.100 & 183.800 & 181.100 & 178.000 & 183.400 \\
Baleares & 169.900 & 158.700 & 160.600 & 173.000 & 197.800 \\
Canarias & 326.800 & 325.500 & 323.600 & 344.800 & 350.300 \\
Cantabria & 88.900 & 90.100 & 90.500 & 89.100 & 88.000 \\
Castilla-León & 433.500 & 427.800 & 429.400 & 413.300 & 423.100 \\
Castilla-La Mancha & 247.400 & 246.100 & 242.900 & 241.200 & 241.800 \\
Cataluña & 1.195 .300 & 1.187 .700 & 1.164 .100 & 1.215 .900 & 1.261 .700 \\
Valencia & 693.400 & 698.300 & 684.100 & 675.400 & 708.800 \\
Extremadura & 161.200 & 165.700 & 163.600 & 157.900 & 155.800 \\
Galicia & 434.600 & 434.200 & 433.100 & 431.600 & 427.100 \\
Madrid & 1.149 .800 & 1.167 .400 & 1.127 .200 & 1.114 .400 & 1.188 .000 \\
Murcia & 170.800 & 166.500 & 170.200 & 169.900 & 177.600 \\
Navarra & 95.100 & 90.600 & 90.200 & 90.000 & 95.300 \\
País Vasco & 392.100 & 388.000 & 383.000 & 393.600 & 396.700 \\
La Rioja & 42.500 & 40.900 & 41.400 & 39.800 & 40.500 \\
\hline
\end{tabular}

Fuente: INE. EPA 1991-1995. 
MAPA 7. Empleados en hoteles sobre el total de ocupados 1994 (\%).

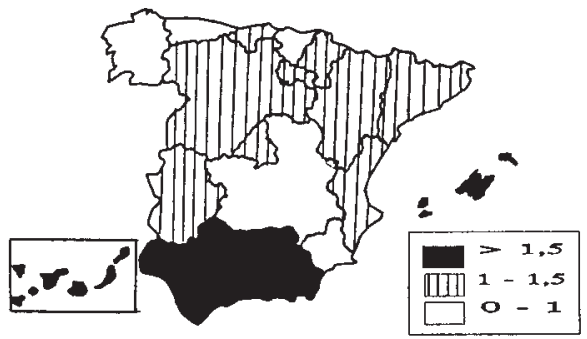

Fuente: INE. MVEH 1994. Elaboración propia.

MAPA 8. Incremento del personal empleado en hoteles 1991-1994 (\%).

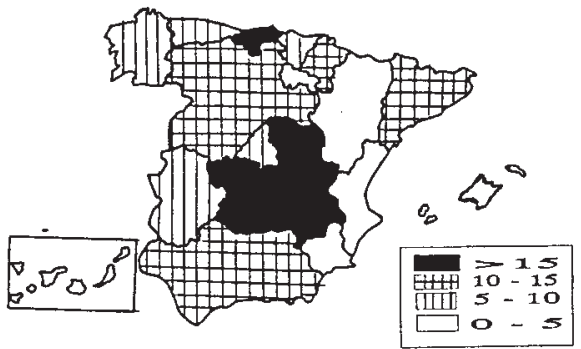

Fuente: INE. MVEH 1991-1994. Elaboración propia.

MAPA 9. Empleados fijos en establecimiento hoteleros 1994.

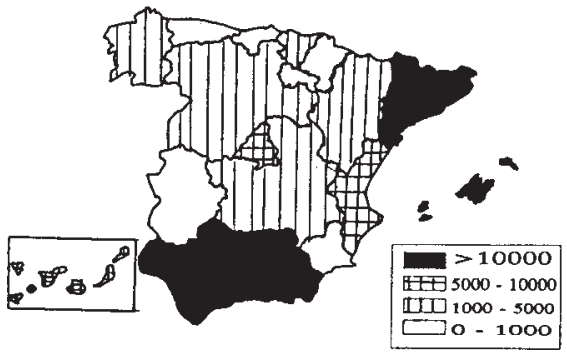

Fuente: INE. MVEH 1994. Elaboración propia.
Mapa 10. Personal eventual en establecimientos hoteleros 1994.

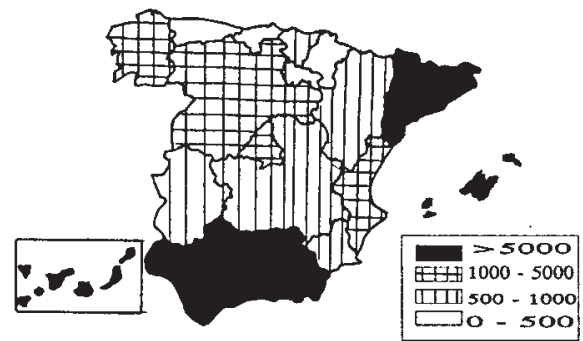

Fuente: INE. MVEH 1994. Elaboración propia.

Mapa 11. Personal fijo sobre el total de ocupados en hoteles 1994 (\%).

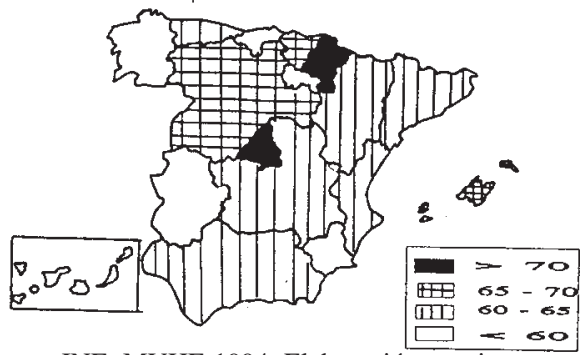

Fuente: INE. MVHE 1994. Elaboración propia.

MAPa 12. Personal eventual sobre el total de ocupados en hoteles 1994 (\%).

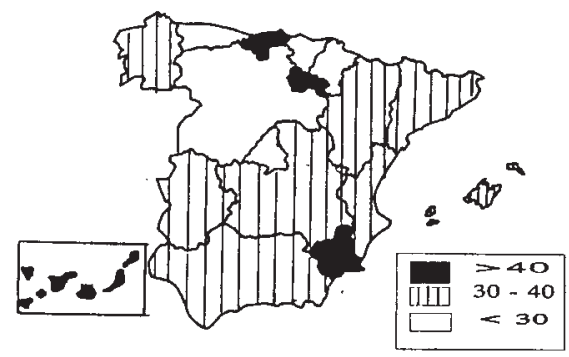

Fuente: INE. MVEH 1994. Elaboración propia.

Este conjunto de cifras no hace más que recalcar el hecho ya conocido de la magnitud de la actividad y el empleo turísticos en determinados espacios regionales españoles (fundamentalmente las islas y las regiones mediterráneas), pero haciendo una distinción entre aquellas Comunidades en las que el sector tiene una trascendencia indiscutible (caso de Baleares, Canarias y, en cierta medida, Andalucía) y aquellas otras en las que su peso relativo desciende si comparamos la ocupación laboral en hotelería con el resto de subsectores de los servicios ${ }^{31}$ (casos de Cataluña, Madrid o Valencia).

31 En los casos de Valencia o Cataluña, regiones consideradas como turísticas debido a la entidad económica de la actividad, los empleados en hotelería sólo suponen el 1,16\% y el 1,31\% respectivamente sobre el total de ocupados en el sector servicios. Esto se explica por la magnitud del empleo en los servicios en general y por la importancia de la actividad y la oferta extrahoteleras en estas Comunidades. 

$1995(\%)$

\begin{tabular}{|lc|}
\hline & $\mathbf{1 9 9 5}$ \\
\hline Total & 60,7 \\
Andalucía & 65,2 \\
Aragón & 55,6 \\
Asturias & 58,9 \\
Baleares & 74,9 \\
Canarias & 74,2 \\
Cantabria & 56,2 \\
Castilla-León & 56,1 \\
Castilla-La Mancha & 51,6 \\
Cataluña & 59,3 \\
Valencia & 55,8 \\
Extremadura & 58,2 \\
Galicia & 46,5 \\
Madrid & 73,2 \\
Murcia & 56 \\
Navarra & 53 \\
País Vasco & 59,2 \\
La Rioja & 47,8 \\
\hline
\end{tabular}

Fuente: INE. EPA 1995.

Cuadro 12

EVOLUCIÓN DEL PERSONAL EMPLEADO EN ESTABLECIMIENTOS HOTELEROS ENTRE 1991 Y 1995*

\begin{tabular}{|c|c|c|c|c|c|}
\hline & 1991 & 1992 & 1993 & 1994 & 1995 \\
\hline Total & 104.752 & 110.221 & 99.032 & 111.795 & \\
\hline Andalucía & 16.232 & 18.476 & 17.638 & 17.856 & 18.002 \\
\hline Aragón & 2.792 & 2.817 & 2.848 & 2.812 & \\
\hline Asturias & 1.190 & 1.272 & 1.336 & 1.280 & \\
\hline Baleares & 20.343 & 20.989 & 20.067 & 20.477 & 20.735 \\
\hline Canarias & 15.250 & 15.484 & 15.784 & 15.930 & 15.963 \\
\hline Cantabria & 989 & 1.024 & 1.063 & 1.231 & \\
\hline Castilla-La Mancha & 2.021 & 2.230 & 2.256 & 2.393 & \\
\hline Castilla-León & 4.107 & 4.226 & 4.401 & 4.592 & \\
\hline Cataluña & 14.321 & 15.078 & 15.669 & 15.918 & 14.986 \\
\hline Valencia & 7.612 & 7.909 & 7.534 & 8.109 & 8.188 \\
\hline Extremadura & 1.418 & 1.496 & 1.528 & 1.534 & \\
\hline Galicia & 3.465 & 3.533 & 3.612 & 3.752 & \\
\hline Madrid & 10.076 & 10.593 & 10.143 & 10.590 & 10.175 \\
\hline Murcia & 1.416 & 1.462 & 1.479 & 1.542 & \\
\hline Navarra & 848 & 879 & 908 & 936 & \\
\hline País Vasco & 2.111 & 2.132 & 2.164 & 2.261 & \\
\hline La Rioja & 561 & 621 & 602 & 582 & \\
\hline
\end{tabular}

Fuente: INE. MVEH 1991-1995.

* Para el año 1995 sólo disponemos de los datos de las principales CC.AA. con peso turístico. 
El resto de las regiones, 14 en total, sólo supone el 30\% del empleo hotelero, con numerosas Comunidades en las que significa menos del 1\% del empleo en servicios (caso de Asturias, Castilla-La Mancha, Castilla-León, Galicia, País Vasco, Madrid, Extremadura y Murcia).

El comentario se complica si analizamos la variación del empleo hotelero entre los años 1991 y 1994, ya que entonces se observan varios hechos:

— La estabilización del empleo hotelero en algunos espacios turísticos consolidados (Canarias, Baleares y Valencia).

- El crecimiento de este tipo de empleo en otros espacios turísticos tradicionales (Cataluña y Andalucía) y en nuevas regiones emergentes (Navarra y Castilla-León).

- El gran incremento del empleo hotelero en CC.AA. que apuestan por el turismo como vía de diversificación productiva, propiciando un importante tirón de la actividad y el empleo, aunque en términos absolutos sean cifras exiguas (Castilla-La Mancha y Cantabria).

La gran conclusión es que existen unas regiones tradicionalmente turísticas con gran peso absoluto y relativo del empleo hotelero (Baleares, Canarias, Valencia), que mantienen una baja tasa de crecimiento de dicho empleo, por debajo del 5\%, debido a la situación de relativa saturación del mercado de trabajo turístico en ellas.

Frente a esta situación, otras CC.AA. con peso turístico siguen manteniendo altas tasas de creación de empleo (por encima del 10\% entre 1991 y 1994 en Andalucía y Cataluña), que demuestran la capacidad de crecimiento del sector y sus buenas perspectivas de creación de puestos de trabajo de cara al futuro.

Por último aparecen regiones emergentes, que amplían considerablemente sus niveles de empleo en hotelería, por encima del $12 \%$, signo inequívoco de la apuesta y el apoyo al sector turístico en estos espacios (Navarra, Cantabria, Castilla-León, etc.).

En cuanto a la caracterización del empleo, la conclusión principal que se puede deducir del tratamiento de los datos es la inestabilidad del mismo en cuanto a la forma de contratación, hecho que también puede ser interpretado como signo de flexibilidad y ajuste a las señales del mercado y la evolución de la demanda ${ }^{32}$.

El total de empleados fijos en 1994 era de 72.000, aunque hay que señalar que la evolución entre 1991 y 1994 es negativa en muchas de las regiones turísticas (Baleares, Andalucía, Canarias y Madrid) y cuando no, se mantiene estable (Cataluña y Valencia). Los casos en los que las cifras aumentan fuertemente (Navarra y Cantabria) se interpretan como la consolidación de la actividad turística en estos territorios (cuadro 13, mapa 9).

Por el contrario, el empleo eventual evoluciona de manera inversamente proporcional al fijo. La cifra global de 37.500 empleos eventuales en 1994 muestra un crecimiento netamente positivo en el período estudiado (1991-1994). Todas las CC.AA. aumentan considerablemente el número de trabajadores eventuales, a excepción de Navarra y Aragón, lo cual refuerza la hipótesis de inestabilidad del empleo y de mayor flexibilidad o ajuste a las nuevas condiciones del mercado de trabajo (cuadro 14, mapa 10).

Es evidente que la Ley de Reforma del Mercado Laboral, aprobada en el año 1993, ha ofrecido unas nuevas condiciones muy favorables a los empresarios del sector, y de hecho la actividad hotelera se está beneficiando con extraordinaria eficacia de ese nuevo marco sociolaboral y de las nuevas modalidades de contrato que aparecen.

32 Consúltese la tesis doctoral de Salvador Antón Clavé sobre la caracterización de la actividad turística en el litoral de Tarragona, defendida en la Universidad de Tarragona a finales de 1995. 
MAPA 13. Incremento del personal fijo en hoteles entre 1991-1994 (\%).

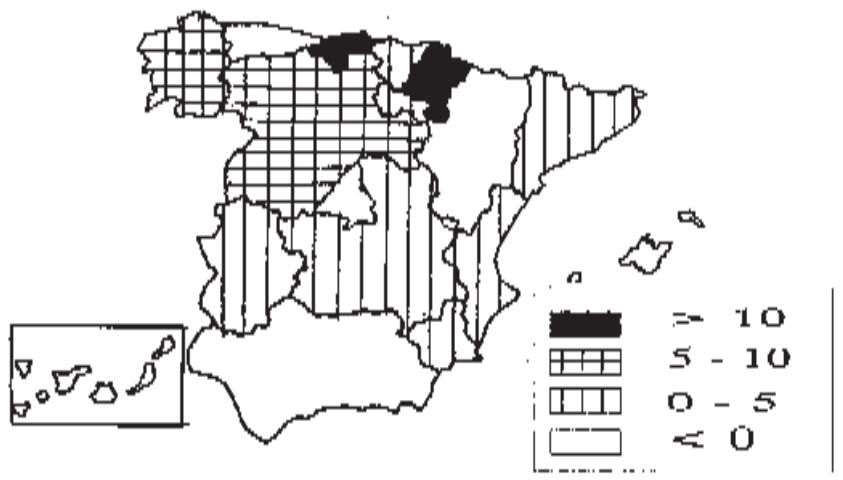

Fuente: INE. MVEH 1991-1994. Elaboración propia.

MAPA 14. Incremento del personal eventual en hoteles entre 1991-1994 (\%).

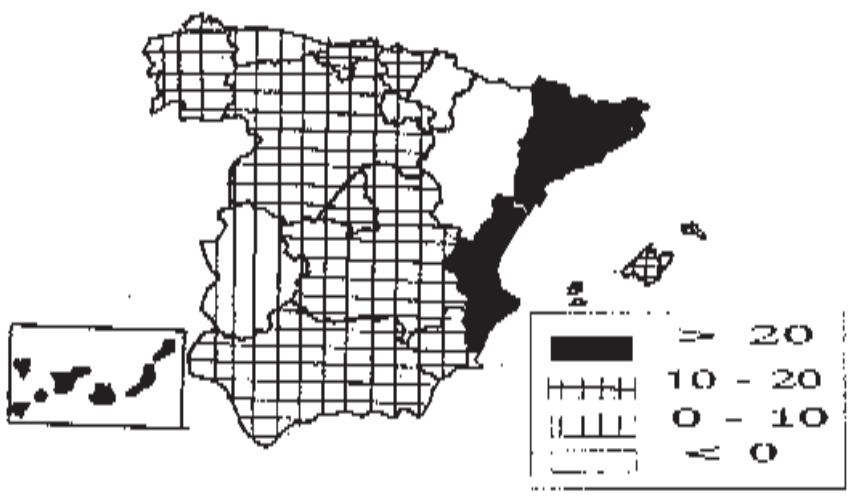

Fuente: INE. MVEH 1991-1994. Elaboración propia.

MaPa 15. Tipos de regiones según características del mercado de trabajo turístico.

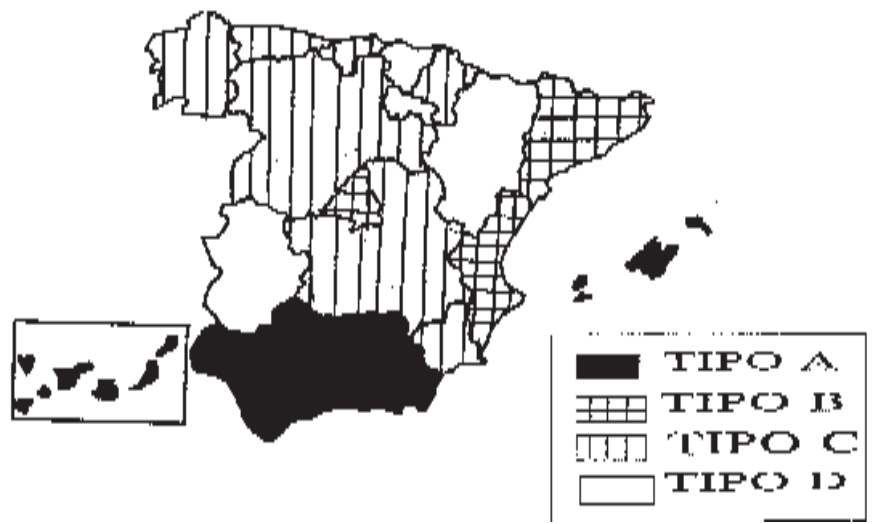

Fuente: Elaboración propia. 
Las conclusiones en materia de empleo hotelero no han de quedarse en el optimismo de las cifras globales, que han aumentado de manera evidente, sino que aparecen nuevas y preocupantes tendencias, como son el inexorable descenso de los trabajadores fijos y el claro aumento de los eventuales ${ }^{33}$.

Las tasas de eventualidad en 1994 nos ofrecen una imagen en la que las regiones tradicionalmente turísticas mantienen un nivel de este tipo de empleo entre el 30 y el $40 \%$ del personal empleado. Sólo Canarias, con un 44,84\%, Murcia y La Rioja, manifiestan una estructura turística extremadamente flexible, en la que el empleo eventual es inherente a la actividad.

Por el contrario, Baleares, Madrid o Navarra, muestran unas tasas de empleo fijo en torno al $70 \%$, lo que implica la consolidación del sector y la menor flexibilidad ante determinadas coyunturas (mapa 11).

El crecimiento del empleo eventual entre 1991 y 1994 es claro en todas las CC.AA., siendo especialmente destacable en Cataluña, Valencia y Canarias, con cifras entre el 25 y el $30 \%$ de incremento.

Otra característica del empleo hotelero, directamente relacionada con la inestabilidad y la flexibilidad, es la estacionalidad del mismo. En general, en la mayoría de las regiones, los mayores índices de empleo hotelero se dan en los meses de vacaciones veraniegas (julio, agosto y septiembre) y en menor medida en abril, junio u octubre.

En esos meses el empleo aumenta entre un 40 y un $70 \%$, siendo especialmente destacable el caso de Baleares, que aumenta en más de un 500\% el personal empleado en verano respecto al ocupado en enero. También Cataluña triplica sus empleados entre los meses de menor y mayor empleo (enero y julio).

En los casos de Valencia, Andalucía y las Comunidades interiores y cantábricas, la estacionalidad es menor, con unas tasas de crecimiento del 50\% entre los meses extremos, lo cual supone un mejor posicionamiento de estas regiones ante el problema de la concentración temporal de la actividad y sus repercusiones sobre la estructura económica y el empleo.

En el otro extremo se encuentran las regiones de Madrid y Canarias, con una tendencia clara de regularidad temporal de la actividad a lo largo de los meses del año. El caso de Canarias se explica por sus particularidades climáticas y el perfil de la demanda, mientras que Madrid presenta una actividad turística sin grandes oscilaciones mensuales por razones de tipología turística y de ubicación geográfica.

En definitiva, es el de la estacionalidad un importante problema del empleo turístico, fruto del cual resultan las características de inestabilidad y eventualidad del mismo. La reducción de esas tasas de estacionalidad es uno de los principales retos para las políticas regionales sobre la actividad turística ${ }^{34}$.

De la minimización de los efectos de esta circunstancia y de la complementariedad del empleo turístico con empleos de otra naturaleza dependerán en buena medida las consecuencias positivas que este sector puede tener sobre los mercados de trabajo regionales.

33 Véanse mapas 13 y 14. En el período que va desde 1991 a 1994 se pasa de 73.062 a 71.136 trabajadores fijos, mientras que los eventuales aumentan de 31.912 a 37.434. Las regiones donde esta tendencia se presenta de forma más acusada son las tradicionalmente turísticas (Canarias, Baleares, Valencia, Cataluña y Andalucía).

34 Op. cit. MARCHENA, M. y VERA, F., 1995. 
Cuadro 13

EVOLUCIÓN DEL PERSONAL FIJO ENTRE LOS AÑOS 1991 Y 1994*

\begin{tabular}{|lrrrr|}
\hline & $\mathbf{1 9 9 1}$ & $\mathbf{1 9 9 2}$ & $\mathbf{1 9 9 3}$ & $\mathbf{1 9 9 4}$ \\
\hline Total & 73.062 & 73.448 & 71.865 & 71.136 \\
Andalucía & 11.766 & 11.824 & 11.525 & 11.245 \\
Aragón & 1.812 & 1.806 & 1.810 & 1.786 \\
Asturias & 857 & 874 & 851 & 828 \\
Baleares & 15.467 & 15.473 & 14.536 & 13.978 \\
Canarias & 9.563 & 9.504 & 9.506 & 8.698 \\
Cantabria & 538 & 546 & 542 & 661 \\
Castilla-La Mancha & 1.460 & 1.448 & 1.453 & 1.527 \\
Castilla-León & 2.821 & 2.872 & 2.910 & 2.989 \\
Cataluña & 10.166 & 10.196 & 10.124 & 10.171 \\
Valencia & 4.721 & 4.726 & 4.294 & 5.010 \\
Extremadura & 862 & 908 & 946 & 901 \\
Galicia & 1.974 & 1.964 & 2.058 & 2.102 \\
Madrid & 8.012 & 8.149 & 8.098 & 7.939 \\
Murcia & 818 & 854 & 890 & 820 \\
Navarra & 536 & 545 & 587 & 717 \\
País Vasco & 1.421 & 1.476 & 1.454 & 1.483 \\
La Rioja & 268 & 283 & 281 & 281 \\
\hline
\end{tabular}

Fuente: INE. MVEH 1991-1994.

* No disponemos todavía de datos sobre personal fijo para el año 1995.

Cuadro 14

EVOLUCIÓN DEL PERSONAL EVENTUAL EN ESTABLECIMIENTOS HOTELEROS ENTRE 1991 Y 1994*

\begin{tabular}{|lrrrr|}
\hline & $\mathbf{1 9 9 1}$ & $\mathbf{1 9 9 2}$ & $\mathbf{1 9 9 3}$ & $\mathbf{1 9 9 4}$ \\
\hline Total & 31.912 & 34.101 & 34.214 & 37.434 \\
Andalucía & 5.363 & 6.164 & 5.545 & 5.984 \\
Aragón & 931 & 962 & 957 & 917 \\
Asturias & 354 & 381 & 378 & 356 \\
Baleares & 5.221 & 5.394 & 5.326 & 6.226 \\
Canarias & 5.842 & 5.913 & 5.940 & 7.144 \\
Cantabria & 448 & 463 & 467 & 494 \\
Castilla-La Mancha & 628 & 632 & 646 & 745 \\
Castilla-León & 1.179 & 1.228 & 1.234 & 1.325 \\
Cataluña & 3.894 & 4.291 & 4.918 & 5.112 \\
Valencia & 2.685 & 2.955 & 3.027 & 3099 \\
Extremadura & 511 & 542 & 528 & 531 \\
Galicia & 1.127 & 1.208 & 1.211 & 1.306 \\
Madrid & 2.034 & 2.196 & 2.209 & 2.357 \\
Murcia & 582 & 612 & 629 & 667 \\
Navarra & 268 & 287 & 299 & 222 \\
País Vasco & 584 & 601 & 616 & 675 \\
La Rioja & 261 & 272 & 284 & 274 \\
\hline
\end{tabular}

Fuente: INE. MVEH 1991-1994.

* No disponemos todavía de datos sobre personal eventual para el año 1995. 


\section{Conclusiones}

Desde el punto de vista general planteamos tres conclusiones acerca de las características y las tendencias del empleo turístico ${ }^{35}$ en España:

A. El sector turístico ha sido el sostén de creación de empleo que ha minimizado los efectos negativos que la crisis de principios de los 90 ha tenido para el mercado de trabajo. El volumen y los ritmos de crecimiento de la ocupación en el sector han sido muy superiores a la media de otras actividades, y la tendencia no se agota, sino que tiene perspectivas de continuar su expansión.

B. Gran parte del empleo creado ha sido de carácter eventual, superando la creación de empleo fijo y muy por encima de los ratios de eventualidad de otras actividades. Se trata de un buen ejemplo de la llamada flexibilidad y de perfecta adecuación a las nuevas características del mercado de trabajo que se pretende por parte de las políticas económicas al uso.

C. Se trata de un empleo que, a pesar de las altas tasas de personal directivo, esconde una descualificación evidente. Junto a ello se observa una baja productividad y un alto grado de sobreexplotación de la mano de obra como nos demuestran los datos sobre horas semanales trabajadas.

Como conclusión de naturaleza territorial, proponemos una clasificación tipológica regional de las CC.AA. españolas en función de las características del mercado de trabajo turístico (mapa 15):

a. Regiones turísticas consolidadas, con fuerte peso del empleo en servicios en general y del turístico en particular, tanto en términos absolutos como relativos, pero en las que se observa una cierta saturación del empleo fijo y un importante aumento del trabajo eventual (Baleares, Canarias y en cierta medida Andalucía). En estas CC.AA. el turismo ha actuado como válvula de escape ocupacional en momentos de crisis (años 1991, 1992 y 1993), propiciando resultados mucho mejores que en el resto de Comunidades.

b. Regiones con peso turístico pero con menor importancia relativa de la actividad. Son Comunidades con grandes montantes de empleo turístico, pero cuya magnitud se diluye dentro de una estructura productiva diversificada, en la que tienen también importante presencia el sector industrial y otros subsectores de servicios avanzados, transportes y comunicaciones, innovación y desarrollo tecnológico, etc. (Cataluña, Madrid y, hasta cierto punto, Valencia). En estas regiones la actividad turística se integra positivamente con el resto de sectores, consolidando un volumen importante de empleo que complementa las posibles contracciones de otras actividades.

c. Regiones que apuestan por el turismo como estrategia de diversificación productiva que minimice los posibles riesgos de la especialización agrícola o el declive industrial (Galicia, Asturias, Cantabria, Navarra, Castilla-León, Castilla-La Mancha, Murcia). Este hecho implica un proceso de diversificación turística y de desa-

35 Hemos seguido en parte algunas reflexiones de MÉNDEZ GUTIÉRREZ DEL VALLE, R.: «Hacia una nueva división del trabajo en España», en Revista de Estudios Regionales, $\mathrm{n}^{\circ}$ 42. Sevilla: Universidades de Andalucía, 1995. 
rrollo de otras tipologías acordes con las nuevas demandas, como es el caso del turismo rural, el turismo urbano, el turismo histórico-cultural o el de naturaleza.

d. Regiones no turísticas, en las que la actividad y el empleo tienen una presencia exigua, y que no muestran signos de desarrollo y expansión del sector (Rioja, Extremadura, País Vasco, Aragón). 